\title{
The Gell-Mann Formula for Representations of Semisimple Groups*
}

\author{
R. Hermann \\ Argonne National Laboratory and Northwestern University \\ Received December 20, 1965
}

\begin{abstract}
A method for constructing representations of non-compact semisimple groups from representations of semidirect product groups is presented. Necessary and sufficient algebraic conditions for the method to work are given, and these are applied to cases of possible interest for the classification of elementary particles.
\end{abstract}

\section{Introduction}

Let $G$ be a non-compact, semisimple Lie group with finite center, with $K$ its maximal compact subgroup. Let $\mathbf{G}$ be its Lie algebra, $\mathbf{G}=\mathbf{K} \oplus \mathbf{P}$ its Cartan decomposition, i.e. $[\mathbf{K}, \mathbf{P}] \subset \mathbf{P},[\mathbf{P}, \mathbf{P}] \subset \mathbf{K}$. Let $\mathbf{G}^{\prime}$ be its contracted Lie algebra: $\mathbf{G}^{\prime}=\mathbf{K} \oplus \mathbf{P}^{\prime}$, isomorphic as a vector space with $\mathbf{G}$, and with the same commutation relations, except that

$$
\left[\mathbf{P}^{\prime}, \mathbf{P}^{\prime}\right]=0 \text {. }
$$

In previous work, we have pointed out that many unitary representations of $G$ can be constructed from unitary representions of $G^{\prime}$ in a simple way. This is important from a practical point of view, since many aspects of the theory of representations are much simpler for groups, such as $G^{\prime}$, which are semidirect products of compact and Abelian groups. For example, the problem of decomposing their tensor products is comparatively easy (e.g., see [3], Chapter 12).

The Gell-Mann formula is a particulary simple method of expressing the operators of $G$ in terms of those of $G^{\prime}$. In [3], Chapter 18, we gave a set of sufficient conditions that the formula hold, based on the geometric properties of the representations of $G^{\prime}$. In this paper we analyze the formula in a more algebraic manner, using Wigner's method [7] for describing the representations of $G^{\prime}$. The results to be obtained below are more complete: again we find that the symmetric spaces $K / L$ on which $G$ also acts transitively play the key role.

\section{Representations of a group in terms of its contracted group}

Let $\mathbf{G}, \mathbf{K}, \mathbf{P}$ and $\mathbf{P}^{\prime}$ be as described above. Let $Z_{u}, 1 \leqq u, v, \ldots \leqq m$, and $X_{i}, 1 \leqq i, j, \ldots \leqq n$, be bases of $\mathbf{K}$ and $\mathbf{P}$, respectively, that are

\footnotetext{
* This work was supported by the Office of Naval Research, \# NONR 3656 (09).
} 
orthonormal with respect to the Killing form of $\mathbf{G}$. Adopt the summation convention on these indices. Write down the structure constants of $\mathbf{G}$ with respect to this basis:
a) $\left[Z_{u}, Z_{v}\right]=c_{u v w} Z_{w}$
b) $\left[Z_{u}, X_{i}\right]=c_{u i j} X_{j}$
c) $\left[X_{i}, X_{j}\right]=c_{i j u} Z_{u}$.

Let $\mathbf{G}^{\prime}=\mathbf{K} \oplus \mathbf{P}^{\prime}$ be the contracted algebra of $\mathbf{G}$. Suppose that $\mathbf{G}^{\prime}$ is realized as a Lie algebra of operators on a Hilbert space $H$ such that the $Z_{u}$ are skew-Hermitian and the $X_{i}^{\prime}$ are Hermitian operators. Suppose that $\left(a_{i u j}\right)$ is a real tensor such that the following formula defines a representation of $\mathbf{G}$ :

$$
X_{i}=a_{i u j} Z_{u} X_{j}^{\prime}
$$

We shall examine in the next section the conditions that the $\left(a_{i u j}\right)$ must satisfy in order that this be a representation of $\mathbf{G}$. In this section we shall assume it is so and deduce the consequences.

First, notice that the $X_{i}$ so defined are not skew-Hermitian. The method of [5], Section 5, can be tried to construct an associated skewHermitian representation of $\mathbf{G}$ and a system of infinitesimal multipliers which will enable the representation to be continued analytically.

Let $X_{i}^{*}$ be the Hermitian adjoint of $X_{i}$. Then,

$$
\begin{aligned}
X_{i}^{*} & =-a_{i u j} X_{j}^{\prime} Z_{u} \\
X_{i}+X_{i}^{*} & =a_{i u j}\left[Z_{u}, X_{j}^{\prime}\right] \\
& =a_{i u j} c_{u j k} X_{k}^{\prime} .
\end{aligned}
$$

The set of operators $X_{i}+X_{i}^{*}$ is then abelian: By the results of Section 5 of [5] we obtain a skew-Hermitian representation of $\mathbf{G}$ by assigning $\frac{1}{2}\left(X_{i}-X_{i}^{*}\right)$ to $X_{i}$, with $Z_{u}$ continuing to define the representation of K. Further, assigning $\frac{1}{2}\left(X_{i}-X_{i}^{*}\right)+\lambda\left(X_{i}+X_{i}^{*}\right)$ to $X_{i}$, with $\lambda$ a fixed complex number, defines an analytic continuation of the representation.

Now, (2.3) can be further simplified under the condition:

$\operatorname{Ad} K$ acting in $\mathbf{P}$ is irreducible.

For, $\operatorname{Ad} K$ acts on the indices $i$ and $u$ via orthogonal transformations, since the bases $\left(X_{i}\right)$ and $\left(Z_{u}\right)$ are orthonormal with respect to the Killing form and $\operatorname{Ad} K$ preserves the Killing form. In order that the $X_{i}$ transform in the same way as the $X_{i}^{\prime}$ under $\operatorname{Ad} K$ the tensor $\left(a_{i u j}\right)$ must be an invariant tensor under the action of $K .\left(c_{u i j}\right)$ is also such an invariant tensor; hence $\left(a_{i u j} c_{u j k}\right)$ is also an invariant tensor. But, using (2.3), the only such invariant tensor is a multiple of the tensor $\delta_{i k}$, i.e. we must have a relation of the form:

$$
a_{i u j} c_{u j k}=\beta \delta_{i k}, \quad \text { with } \beta \text { a real constant. }
$$


Hence,

$$
X_{i}+X_{i}^{*}=\beta X_{i}^{\prime},
$$

i.e. the $X_{i}^{\prime}$ themselves are the multipliers for the representation. Further,

$$
\begin{aligned}
\frac{1}{2}\left(X_{i}-X_{i}^{*}\right) & =\frac{1}{2} a_{i u j}\left(X_{j}^{\prime} Z_{u}+Z_{u} X_{j}^{\prime}\right) \\
& =a_{i u j} X_{j}^{\prime} Z_{u}+\frac{1}{2} a_{i u j}\left[Z_{u}, X_{j}^{\prime}\right] \\
& =a_{i u j} X_{j}^{\prime} Z_{u}+\frac{1}{2} a_{i u j} c_{u j k} X_{k}^{\prime} \\
& =a_{i u j} X_{j}^{\prime} Z_{u}+\frac{1}{2} \beta X_{i}^{\prime} .
\end{aligned}
$$

One obvious candidate for the tensor $\left(a_{i u j}\right)$ would be the tensor $\left(c_{u i j}\right)$. Now, with this choice,

$$
X_{i}=c_{u i j} X_{j}^{\prime} Z_{u}=\left[Z_{u}, X_{i}^{\prime}\right] Z_{u} .
$$

Let $\Delta=Z_{u} Z_{u}$ be the Casimir operator of $K$. We see that this choice of $X_{i}$ leads to the "Gell-Mann formula" type of representation of $\mathbf{G}$, i.e.

$$
X_{i} \rightarrow\left[\Delta, X_{i}^{\prime}\right]+\lambda X_{i}^{\prime} .
$$

Conditions that this actually be a representation of $G$ have been given in [3]. Further investigations follow.

\section{Conditions for the validity of the Gell-Mann formula}

Suppose that (2.2) really does define a representation of $\mathbf{G}$. We want to derive the necessary and sufficient algebraic conditions on the tensor $\left(a_{i u j}\right)$.

The first condition is that the tensor be invariant under $K$. As we have seen, this leads to (2.5), if (2.4) is satisfied. Now, (2.1 c) must bc satisfied:

$$
\begin{aligned}
c_{i k u} Z_{u} & =\left[X_{i}, X_{k}\right] \\
& =a_{i u j} a_{k v h}\left[Z_{u} X_{j}^{\prime}, Z_{v} X_{h}^{\prime}\right] \\
& =a_{i u j} a_{l v h}\left(Z_{u} X_{j}^{\prime} Z_{v} X_{h}^{\prime}-Z_{v} X_{h}^{\prime} Z_{u} X_{j}^{\prime}\right) \\
& =a_{i u j} a_{k v h}\left(Z_{u} Z_{v} X_{j}^{\prime} X_{h}^{\prime}+Z_{u}\left[X_{j}^{\prime}, Z_{v}\right] X_{h}^{\prime}-Z_{v} Z_{u} X_{h}^{\prime} X_{j}^{\prime}-\right. \\
& \left.-Z_{v}\left[X_{h}^{\prime}, Z_{u}\right] X_{j}^{\prime}\right) \\
& =a_{i u j} a_{k v h}\left(\left[Z_{u}, Z_{v}\right] X_{j}^{\prime} X_{h}^{\prime}-Z_{u} c_{v j l} X_{l}^{\prime} X_{h}^{\prime}+Z_{v} c_{u h l} X_{l}^{\prime} X_{j}^{\prime}\right) .
\end{aligned}
$$

Now let us apply both sides of this identity to a properly chosen vector $\psi^{0}$. It is most convenient to choose $\psi^{0}$ as an eigenvector of the operators $X_{i}^{\prime}$, i.e.

$$
X_{i}^{\prime}\left(\psi^{0}\right)=p_{i}^{0} \psi^{0}
$$

(Since the operators $X_{i}^{\prime}$ have continuous spectrum this requires that the eigenvectors be "outside" the Hilbert space, e.g. "generalized eigenvectors" in the sense of GELFAND and VILENkin [1]. Here, we are following Wigner's method for describing representations of $G^{\prime}[7]$ ). 
The adjoint action of $K$ on $\mathbf{P}^{\prime}$ defines an action of $K$ on the vectors $\left(p_{i}\right)$ : Let $L$ be the group consisting of those elements of $K$ that leave $\left(p_{i}^{0}\right)$ invariant. $L$ is then what WIGNER calls the "little group". Let $\mathbf{L}$ be the corresponding subalgebra of $\mathbf{K}$. For $\mathbf{Z} \in \mathbf{L}$,

$$
\left(\operatorname{Ad} \operatorname{Exp}(t Z) X_{i}^{\prime}\right)\left(\psi^{0}\right)=p_{i}^{0} \psi^{0} \quad \text { for all } t .
$$

Taking the derivative with respect to $t$ gives:

$$
\left[Z, X_{i}^{\prime}\right]\left(\psi^{0}\right)=0, \quad \text { or } \quad X_{i}^{\prime} Z \psi^{0}=p_{i}^{0} Z \psi^{0},
$$

i.e. $L$ has a representation on the space of eigenvectors of $\mathbf{P}^{\prime}$ with given eigenvalue $p_{i}^{0}$. Conversely, if $Z$ satisfies (3.2), it belongs to $L$. In particular, the set of all $Z$ such that

$Z\left(\psi^{0}\right)=0$ for all eigenvectors $\psi^{0}$ of eigenvalues $p_{i}^{0}$ forms an ideal of $\mathbf{L}$, which we call $\mathbf{L}^{0}$.

Apply $\psi^{0}$ to both sides of (3.1).

$$
c_{i k u} Z_{u}\left(\psi^{0}\right)=a_{i u j} a_{k v h}\left(p_{j}^{0} p_{h}^{0}\left[Z_{u}, Z_{v}\right]-c_{v j l} p_{l}^{0} p_{h}^{0} Z_{u}+c_{u h l} p_{l}^{0} p_{j}^{0} Z_{v}\right)\left(\psi^{0}\right)
$$

hence:

$c_{i k u} Z_{u}-a_{i u j} a_{k v h}\left(p_{j}^{0} p_{h}^{0}\left[Z_{u}, Z_{v}\right]-c_{v j l} p_{l}^{0} p_{h}^{0} Z_{u}+c_{u h \imath} p_{l}^{0} p_{j}^{0} Z_{v}\right) \in \mathbf{L}^{0}$.

Let us put this condition in a form independent of the basis chosen for G. Define

Note that

$$
X^{0}=p_{i}^{0} X_{i}
$$

$Z \in \mathbf{L}$ if and only if $\left[Z, X^{0}\right]=0$ and $Z \in \mathbf{K}$.

Now define $T$ as a bilinear map $\mathbf{P} \times \mathbf{P} \rightarrow \mathbf{K}$ by the formula:

$$
T\left(X_{i}, X_{j}\right)=a_{i u j} Z_{u} .
$$

It is readily calculated that 3.4 takes the form:

$$
\begin{aligned}
{[X, Y]+} & {\left[T\left(X, X^{0}\right), T\left(Y, X^{0}\right)\right]-T\left(X,\left[T\left(Y, X^{0}\right), X^{0}\right]\right) } \\
+ & T\left(Y,\left[X^{0}, T\left(X, X^{0}\right)\right]\right) \in \mathbf{L}^{0} \text { for all } X, Y \in \mathbf{P} .
\end{aligned}
$$

Now, (3.7) is a necessary condition that (2.2) define a representation of $\mathbf{G}$. However, it is also a sufficient condition: By WIGNER's work, if we chose another system of eigenvalues $\left(p_{i}^{1}\right)$ of $\mathbf{P}^{\prime}$, the element $X^{1}=p_{i}^{1} X_{i}$ would be conjugate under $K$ to $X^{0}$. Note that condition (3.7) remains verified if this replacement is made. Thus (3.1) is an identity among the operators on $H$ if (3.7) is true for one eigenvector $\psi^{0}$.

Let us now turn to the study of that $T$ which leads to the Gell-Mann formula, i.e.

$T(X, Y)=c[X, Y]$ for $X, Y \in \mathbf{P}$, where $c$ is a real constant.

(3.7) then takes the form:

$$
\begin{aligned}
{[X, Y] } & +c^{2}\left[\left[X, X^{0}\right],\left[Y, X^{0}\right]\right]-c^{2}\left[X,\left[\left[Y, X^{0}\right], X^{0}\right]\right]+ \\
& +c^{2}\left[Y,\left[X^{0},\left[X, X^{0}\right]\right]\right] \in \mathbf{L}^{0} \text { for all } X, Y \in \mathbf{P} .
\end{aligned}
$$


Let us also suppose that

$\mathbf{L}=\mathbf{L}^{0}$, i.e. the connected component of the little group acts as the identity in the "rest frame".

We shall not pursue here the most general set of conditions for which (3.9) is true, but shall concentrate on what seems to be the simplest situation. Define subspaces $\mathbf{M}, \mathbf{A}, \mathbf{B}$ of $\mathbf{G}$ as follows:

$\mathbf{A}$ is the set of $X \in \mathbf{P}$ with $\left[X, X^{0}\right]=0$.

$\mathbf{M}$ and $\mathbf{B}$ are the orthogonal complement (with respect to the Killing form of $\mathbf{G}$ ) of $\mathbf{L}$ and $\mathbf{A}$ in $\mathbf{K}$ and $\mathbf{P}$ respectively.

Then,

$$
\begin{gathered}
\mathbf{P}=\mathbf{A} \oplus \mathbf{B} ; \mathbf{K}=\mathbf{L} \oplus \mathbf{M} . \\
{\left[X^{0}, \mathbf{B}\right] \subset \mathbf{M} ;\left[X^{0}, \mathbf{M}\right] \subset \mathbf{B} ;\left(\operatorname{Ad} X^{0}\right)^{2}(\mathbf{B}) \subset \mathbf{B} .}
\end{gathered}
$$

$\mathbf{L} \oplus \mathbf{A}$ is the centralizer of $X^{0}$ in $\mathbf{G}$.

Now we can state our main result:

Theorem. Suppose that either:

$\mathbf{L} \oplus \mathbf{A}=\mathbf{S}$ is a symmetric subalgebra of $\mathbf{G}$, and $\mathbf{G}_{u}=\mathbf{K}+i \mathbf{P}$ is simple,

or

AdL acting in $\mathbf{M}$ is irreducible, or

$\mathbf{I}$ is a symmetric subalgebra of $\mathbf{K}$, and $\mathbf{K}$ is simple.

Then, assuming (3.10), (3.9) is satisfied for one choice of c, i. e. the Gell-Mann formula is verified.

Proof. First, assume (3.13). By definition of "symmetric subalgebra", there is an automorphism $s$ of $G$ such that

a) $s^{2}=$ identity,

b) $\mathrm{S}$ is the set of $X \in \mathrm{G}$ such that $s(X)=X$.

Note that the Killing form of $\mathbf{G}$ restricted to $\mathbf{S}$ is non-degenerate, since $\mathbf{S}=\mathbf{S} \cap \mathbf{K} \oplus \mathbf{S} \cap \mathbf{L}$. (This is the key property in this argument.) Hence, $\mathbf{G}$ is the direct sum of $\mathbf{S}$ and its orthogonal complement, i.e. $\mathbf{B}+\mathbf{M}$, which we call $\mathbf{E}$. Since $s$ leaves this orthogonal complement invariant, and $s$ has as eigenvalues only $\pm 1, s$ must be minus the identity on $\mathbf{E}$, i.e.

$$
[\mathbf{E}, \mathbf{E}] \subset \mathbf{S}[\mathbf{S}, \mathbf{E}] \subset \mathbf{E}
$$

This gives the conditions

$$
\begin{gathered}
{[\mathbf{B}, \mathbf{B}] \subset \mathbf{L} ;[\mathbf{M}, \mathbf{M}] \subset \mathbf{L} ;[\mathbf{M}, \mathbf{B}] \subset \mathbf{A} ;} \\
{[\mathbf{L}, \mathbf{B}] \subset \mathbf{B} ;[\mathbf{L}, \mathbf{M}] \subset \mathbf{M} ;} \\
{[\mathbf{A}, \mathbf{B}] \subset \mathbf{M} ;[\mathbf{A}, \mathbf{M}] \subset \mathbf{B} .}
\end{gathered}
$$

(Notice that (3.17) implies (3.16) also.)

Then, (3.10) and (3.17) imply (3.9) in the following cases:

$$
X, Y \in \mathbf{B} ; X, Y \in \mathbf{A} \text {. }
$$


It only remains to verify (3.9) in the case: $X \in \mathbf{A}, Y \in \mathbf{B}$. In this case (3.9) is equivalent to

$$
\left[X, Y-c^{2}\left(\operatorname{Ad} X^{0}\right)^{2}(Y)\right]=0 .
$$

In turn (3.18) is implied by the following condition:

$\left(\operatorname{Ad} X^{0}\right)^{2}$ acting in $B$ has a single eigenvalue (which can be taken as $c^{2}$ ).

Let us now verify (3.19).

Notice that (3.17) implies that $\mathbf{L}+i \mathbf{A}$ is a symmetric subalgebra of the compact real form of $\mathbf{G}, \mathbf{G}_{u}=\mathbf{K}+i \mathbf{P}$. We are assuming that $\mathbf{G}_{u}$ is a simple Lie algebra: This implies that $\operatorname{Ad}(\mathbf{L}+i \mathbf{A})$ acting in $\mathbf{M}+i \mathbf{B}$ is irreducible [2]. Now, $i X^{0}$ belongs to the center of $\mathbf{L}+i \mathbf{A}$; hence $\operatorname{Ad}\left(i X^{0}\right)^{2}$ acting in $\mathbf{M}+i \mathbf{B}$ must commute with $\operatorname{Ad}(\mathbf{L}+i \mathbf{A})$. By Schur's lemma, $\operatorname{Ad}\left(i X^{0}\right)^{2}$ acting in $\mathbf{M}+i \mathbf{B}$ is $-k$, for some constant $k>0$. This implies that $\mathrm{Ad}\left(X^{0}\right)^{2}$ acting in $\mathbf{M} \oplus \mathbf{B}$ is $k$, i.e. $c$ can be chosen as $k$; (3.19), hence also (3.18) is then satisfied, which implies (3.9).

Now assume (3.14) is satisfied: $\operatorname{Ad} X^{0}$ maps $\mathbf{B}$ isomorphically onto $\mathbf{M}$ and commutes with the action of $\mathrm{Ad} \mathbf{L}$. Hence, again $\left(\operatorname{Ad} X^{0}\right)^{2}$ acting in $\mathbf{B}$ must be a constant. Suppose that $W$ is an eigenvector of $\operatorname{Ad} X^{0}$ with eigenvalue $\lambda$. (All eigenvalues of $\mathrm{Ad} X^{0}$ are real numbers.) Write

Then,

$$
W=Z+Y \text {, with } Z \in \mathbf{K}, Y \in \mathbf{P} \text {. }
$$

$$
\left[X^{0}, Z\right]=\lambda Y ;\left[X^{0}, Y\right]=\lambda Z \text {. }
$$

Conversely, such a pair of elements determines an eigenvector of $\operatorname{Ad} X^{0}$. Then, either:

$$
\lambda=0, Y \in \mathbf{A} ; Z \in \mathbf{L} ; \quad \text { or } \quad Y \in \mathbf{B} ; Z \in \mathbf{M} .
$$

In particular, $\operatorname{Ad} X^{0}$ has only one positive eigenvalue. Suppose that $W^{\prime}$ is another eigenvector of $\operatorname{Ad} X^{0}$ for eigenvalue $\lambda^{\prime}$. Then:

$$
\begin{gathered}
{\left[W, W^{\prime}\right]=0 \text { if } \lambda+\lambda^{\prime} \neq 0} \\
{\left[W, W^{\prime}\right] \in \mathbf{L} \oplus \mathbf{A} \text { if } \lambda=-\lambda^{\prime} .}
\end{gathered}
$$

Writing out these relations gives (3.17), hence proves (3.9).

That (3.15) implies (3.14) is a well-known fact in the theory of symmetric spaces [2]. This finishes the proof of the Theorem.

\section{Remarks}

The fact that $\mathbf{K}$ is a compact subalgebra of $\mathbf{G}$ has not been used very much; one would expect that some of the results carry over if $\mathbf{K}$ is a general symmetric subalgebra of $\mathbf{G}$. This will be considered in a later paper. For example, carrying this over successfully would give a "GellMann formula" for representations of $S O(4,1)$ (the de Sitter group) in terms of representations of its contraction, the Poincaré group. 
In order that a given subgroup $L$ of $K$ satisfy these conditions, it does not just suffice that $L$ be a symmetric subgroup of $K$. In fact the existence of such an $L$ is a restrictive condition on the symmetric space $G / K$. For example, if $K$ has a non-discrete center, i.e. if $G / K$ is a symmetric bounded complex domain, then the domain is a special type called the "Siegel domains of type I". In this case the symmetric space $K / L$ occurs on the boundary of the domain as the "Silov" or "distinguished" boundary. (Physicists are used to this as the part of the boundary over which one must integrate for dispersion relations.) Further, these domains also admit representations as "tube domains", generalizations of the alternate realization of the inside of the unit circle in the complex plane $(=S L(2, R) / S O(2, R))$ as the upper half plane. See [6] for further details about these ideas.

\section{Construction of discrete series representations using the Gell-Mann formula} tions:

Suppose that $\mathbf{G}=\mathbf{K} \oplus \mathbf{P}$ is as before, with the additional assump-

a) $\mathbf{G}$ is a simple Lie algebra.

b) $\mathbf{K}$ has a one-dimensional center; say, it is the multiple of an element $Z^{0} \in \mathbf{K}$.

$\operatorname{Ad} Z^{0}$ acts on $P$ and commutes with the irreducible action there of Ad $K$. Hence, $Z^{0}$ can be normalized so that:

$\left(\mathrm{Ad} Z^{0}\right)^{2}$ acting on $\mathbf{P}$ is minus the identity.

Then, the subset $\left\{X+i\left[Z^{0}, X\right]: X \in \mathbf{P}\right\}=\mathbf{P}^{-}$of the complexification of $\mathbf{G}$ is an abelian subalgebra. A representation of $\mathbf{G}$ by operators on a Hilbert space $H$ is said to be of discrete series type if there is a vector in $H$ which is annihilated by $\mathbf{K}_{S}$ and the abelian algebra $\mathbf{P}^{-}$. $\left(\mathbf{K}_{S}\right.$ is the maximalsemisimple ideal of $\mathbf{K}$, which is a direct sum of $\mathbf{K}_{S}$ and the center $Z^{0}$.)

We will construct discrete series representations in the following way: Start off with a representation of $G^{\prime}$ by operators on a Hilbert space $H$ such that (3.10) and (3.13) are satisfied, and $\mathbf{K}$ has an invariant vector. Then, the Gell-Mann formula applies (but this representation is not of the discrete series type). However, the representation of $\mathbf{G}^{\prime}$ can be modified in the following way:

$Z^{0} \rightarrow \mathbf{Z}^{0}+i b$, with $b$ a real constant. (However, only certain discrete values of $b$ are allowed if the representation is to arise from a representation of the global group $\left.G^{\prime}\right) \mathbf{K}_{S}$ and $\mathbf{P}^{\prime}$ remain unchanged.

We will now show that $\mathbf{L}$ remains unchanged under this modification. (However, at the group level this is not true, since $L$, the centralizer of $X^{0}$ in $K$, is not connected.) This will prove that the Gell-Mann formula remains true for the modfied representation. 
We are given that $\mathbf{L}$ is a symmetric subalgebra of $\mathbf{K}$. Let $s^{\prime}$ be an automorphism of order two of $\mathbf{K}$ which has $\mathbf{L}$ as its set of fixed points and $\mathbf{M}$ as its eigenvectors with eigenvalue -1 . Since $s^{\prime}$ is an automorphism, it must preserve the center and maximal semisimple ideal of $K$. Then,

$$
\begin{aligned}
& s^{\prime}\left(Z^{0}\right)= \pm Z^{0} \\
& s\left(\mathbf{K}_{S}\right)=\mathbf{K}_{S} .
\end{aligned}
$$

$s^{\prime}\left(Z^{0}\right)=Z^{0}$ is impossible, for then $Z^{0} \in \mathbf{L}$, hence $\left[Z^{0}, X^{0}\right]=0$. Thus

$$
Z^{0} \in \mathbf{M} \text {. }
$$

Since $\mathbf{Z}^{0}$ is the only element of $\mathbf{K}$ that is modified, we see that the conditions for the Gell-Mann formula remain verified.

Now, let us compute $X$, for $X \in \mathbf{P}$, as given via the Gell-Mann formula by the modified representation. (4.3) implies that $\mathbf{L} \subset \mathbf{K}_{S}$ and that $\mathbf{M}$ is a direct sum of its intersection with $\mathbf{K}_{S}$ and $Z^{0}$, which are also orthogonal. This implies that the orthonormal basis $\left(Z_{u}\right)$ of $K$ can be chosen so that $Z_{1}$ is the center, while $Z_{u} \in \mathbf{K}_{S}$ for $u>1$. The Casimir operator of $\mathbf{K}$ in the modified representation is then

$$
\left(Z_{1}+i b\right)^{2}+\sum_{u>1} Z_{u}^{2}=\Delta+2 i b Z_{1}-b^{2} .
$$

$X$ is now given by:

$$
X=\left[\Delta, X^{\prime}\right]+2 i b\left[Z_{1}, X^{\prime}\right]+\lambda X^{\prime} .
$$

(In using this formula, care must be taken with its implicit assumptions: $\Delta$ is the Casimir operator of $K$ in the original representation of $\mathbf{G}^{\prime}$, and $Z_{1}$ is the element in the center of $K$ whose length in the Killing metric is - 1.)

Now, let us suppose that $\psi^{\prime}$ is a vector of $H$ which is annihilated by $K$ in the original representation of $G^{\prime}$. We shall show that, by a proper choice of $\lambda$ (depending on $b$, of course) $\psi^{\prime}$ can be annihilated by $\mathbf{P}^{-}=\left\{X+i\left[Z^{0}, X\right]: X \in \mathbf{P}\right\}$. From 4.6, we have:

$X+i\left[Z^{0}, X\right]+\left[\Delta, X^{\prime}+i\left[Z^{0}, X^{\prime}\right]\right]+(\lambda+2 a b)\left(X^{\prime}+i\left[Z^{0}, X^{\prime}\right]\right)$,

where $a$ is the constant such that $Z_{1}=a Z^{0}$.

Since $\Delta$ is the Casimir operator of $K$ in the unmodified representation,

$$
\Delta \psi^{\prime}=0 \text {. }
$$

The subspace of $H$ spanned by vectors of the form

$$
\left.\left\{X^{\prime}+i\left[Z^{0}, X^{\prime}\right]\right) \psi^{\prime}: X^{\prime} \in \mathbf{P}^{\prime}\right\}
$$

is invariant and irreducible under $K$. Hence, $\Delta$ has a constant value in this subspace, say $\delta$, which only depends on the way $\operatorname{Ad} K$ acts in $\mathbf{P}$. Then,

$$
\left(X+i\left[Z^{0}, X\right]\right) \psi^{\prime}=(\delta+\lambda+2 a b)\left(X^{\prime}+i\left[Z^{0}, X^{\prime}\right]\right) \psi^{\prime} .
$$


Then, for $\lambda=-2 a b-\delta$, we see that $\mathbf{P}^{-}$annihilates $\psi^{\prime}$, hence the representation of $G$ is of the "discrete series" type. Of course, it is not, as it stands, skew-Hermitian, since $\lambda$ is real. This can be handled by modifying the Hilbert space inner product of the subspace of $H$ generated by the operators of $G$ applied to $\psi^{\prime}$, following the pattern described in Section 6 of [5] for the case $G=S L(2, R)$. (In fact, notice that all of the work in this section is modelled after the calculations made in [5] for this case.) We might mention without proof the geometric meaning of this construction. $K$ acts via the adjoint representation in P. $L$ is the isotropy subgroup of $K$ at the point $X^{\mathbf{0}} \in \mathbf{P}$, hence the orbit of $K$ at $X^{0}$ is just $K / L$, i.e. the homogeneous space $K / L$ is a submanifold of $P$ passing through the point $X^{0}$. Further, $\mathbf{P}$ can be made into a complex vector space. It can be proved $[2,6]$ that the homogeneous space $G / K$ can be realized as a bounded domain $D$ in $\mathbf{P}$ having the orbit $K / L$ on the boundary as one of the orbits of $G$. (In fact, this is the only compact orbit of $G$ on the boundary.) One can consider a complex analytic line bundle on $G / K$. Consider the space of cross-sections of this bundle that are complex analytic and that can be extended smoothly to the boundary of $D$. $G$ will admit a representation on this space of cross-sections (assuming, of course, that the line bundle is invariant under the action of $G$ ), and this representation can be made unitary by a proper choice of multipliers. However, the cross-sections will also have "boundary values" as functions on $K / L$, which, we recall, lies on the boundary of $D$. If we realize $H$ as a space of functions on $K / L$, we recognize that we have picked out certain subspaces of $H$ that in a certain sense are "boundary values" of analytic cross-sections of complex analytic line bundles on $D=G \mid K$. Again, these remarks will clear if the reader considers the case where $D=$ (the inside of the unit circle in the complex plane), $K \mid L=$ (the boundary).

As an interesting example to which this theory applies and which is of interest for physical applications, we can consider the case $G=S U(n, n)$, which was briefly treated by the geometric method in [4]. Here we shall emphasize the constructions which show that the hypotheses of Theorem 3.1 are satisfied.

Let $V$ be an $n$ dimensional complex Hilbert space, with inner product denoted by $\left\langle v \mid v^{\prime}\right\rangle$. Let $V^{\prime}$ be the direct sum $V \oplus V$ of two copies of $V$, with the following Hermitian inner product:

$$
\left\langle v_{1} \oplus v_{2} \mid v_{1}^{\prime} \oplus v_{2}^{\prime}\right\rangle=\left\langle v_{1} \mid v_{1}^{\prime}\right\rangle-\left\langle v_{2} \mid v_{2}^{\prime}\right\rangle .
$$

This inner product has $n$ plus and $n$ minus signs; its group of unitary transformations is $U(n, n)$. (By a "unitary" or "Hermitian" operator, we mean relative the indefinite metric (4.9) on $V^{\prime}$. The adjoint relative to (4.9) is denoted by *. The Lie algebra of $S U(n, n)$ consists of those 
skew-Hermitian transformations on $V^{\prime}$ with trace zero. Consider the following unitary transformation:

$$
g_{0}\left(v_{1} \oplus v_{2}\right)=v_{1} \oplus-v_{2} .
$$

Then, $g_{0}^{2}=$ identity. The subgroup of $U(n, n)$ that commutes with $g_{0}$ is then $U(n) \times U(n)$, i.e. the transformations that preserve the subspaces $V \oplus(0)$ and $(0) \oplus V$. It is a maximal compact subgroup. Consider the following operator on $V^{\prime}$ :

$$
X^{0}\left(v_{1} \oplus v_{2}\right)=-v_{2} \oplus v_{1} .
$$

$i X^{0}$ is skew-Hermitian, has zero trace, hences belongs to the Lie algebra of $S U(n, n) . g \in U(n) \times U(n)$ commutes with $X^{0}$ if and only if $g$ belongs to the diagonal subgroup of $U(n) \times U(n)$. Let us prove this:

Suppose $g\left(v_{1} \oplus v_{2}\right)=g_{1} v_{1} \oplus g_{2} v_{2}$, and $g X^{0}=X^{0} g$. Then,

$$
\begin{aligned}
X^{0} g\left(v_{1} \oplus v_{2}\right) & =X^{0}\left(g_{1} v_{1} \oplus g_{2} v_{2}\right)=-g_{2} v_{2} \oplus g_{1} v_{1} \\
g X^{0}\left(v_{1} \oplus v_{2}\right) & =g\left(-v_{2} \oplus v_{1}\right) \\
& =-g_{1} v_{2} \oplus g_{2} v_{1},
\end{aligned}
$$

hence $g_{1}=g_{2}$, i.e. $g$ belongs to the diagonal subgroup.

Now, $X_{0}^{2}=-$ identity. $\operatorname{Ad} g_{0}\left(X^{0}\right)=-X^{0}$, i.e. $X^{0} \in \mathbf{P}$. Suppose that $X$ is a skew-Hermitian operator on $V^{\prime}$. Then,

$$
X^{0} X X^{0-1} \text { is also skew-Hermitian. }
$$

(Proof: $\left(X^{0} X X^{0-1}\right)^{*}=-X^{0-1} X^{*} X^{0}=-X^{0} X^{*} X^{0-1}$.)

Hence, $X \rightarrow X^{0} X X^{0-1}$ defines an automorphism of the Lie algebra of $S U(n, n)$ of order two, i.e. the centralizer of $X^{0}$ in the Lie algebra of $S U(n, n)$ is a symmetric subalgebra, as required to verify the hypotheses of the Theorem. In fact $\left(\operatorname{Ad} i X^{0}\right)^{2}$ is the identity, so that (3.19) is automatically satisfied.

\section{Bibliography}

[1] Gel'fand, I. M., and N. YA. VILENkIN: Generalized functions, vol. 4. New York: Academic Press 1964.

[2] Helgason, S.: Differential geometry and symmetric spaces. New York: Academic Press 1962.

[3] Hermann, R.: Lie groups for physicists. New York: W. A. Benjamin Inc. 1965.

[4] - Some properties of representations of non-compact groups, to appear. Proceedings of the Seminar on Elementary Particle Physics, International Center for Theoretical Physics, Trieste, 1965

[5] - Analytic continuation of group representation, to appear.

[6] Koranyi, A., and J. A. WoLF: Realizations of hermitian symmetric spaces as generalized half-planes. Ann. Math. 8, 265-288 (1965).

[7] WIGNER, E.: Unitary representations of the Lorentz group. Ann. Math. 40, 149 (1939). 